\title{
Peritoneal strumosis, 10 years after laparoscopic surgery for mature cystic teratoma of the ovary: a case report
}

\author{
Jun-ichi Akahira $\cdot$ Mareyuki Endo $\cdot$ \\ Ryoji Chiba · Koji Tanoguchi · Jun-ichiro Yamauchi • \\ Shu-ichi Ishiyama $\cdot$ Taiichiro Meguro
}

Received: 22 February 2013/Accepted: 25 March 2013/Published online: 14 April 2013

(C) The Japan Society of Clinical Oncology 2013

\begin{abstract}
Peritoneal strumosis, a condition in which benign thyroid tissue spreads to the peritoneal cavity, is extremely rare. Here we report the first case of peritoneal strumosis, 10 years after laparoscopic surgery for mature cystic teratoma of the ovary. A 64-year-old woman was referred to our hospital due to suspicion of a recurrence of rectal carcinoma in September 2011. Under the diagnosis of recurrence and peritoneal dissemination of rectal carcinoma, she underwent laparoscopic surgery in October 2011. On laparoscopy, several small whitish nodules on the peritoneum and mesentery, diagnosed as benign thyroid tissue, were identified. After surgery, re-examination of her past history revealed that she had received a laparoscopic salpingo-oophorectomy for a right ovarian tumor at another hospital, 10 years prior. In the surgical record, spillage of fatty component was described during the laparoscopic surgery. Pathological diagnosis of mature cystic teratoma with a component of thyroid tissue had been made. Although rare, peritoneal strumosis must be kept in mind in the differential diagnosis of late complications after laparoscopic surgery for mature cystic teratoma of the ovary.
\end{abstract}

Keywords Laparoscopy - Mature cystic teratoma . Ovarian tumor - Peritoneal strumosis - Thyroid gland

J. Akahira $(\bowtie) \cdot$ M. Endo · R. Chiba · J. Yamauchi ·

$\mathrm{S}$. Ishiyama $\cdot \mathrm{T}$. Meguro

Department of Pathology and Surgery, Sendai Kousei Hospital,

4-15 Hirose-machi, Aoba-Ku,

Sendai 980-0873, Japan

e-mail: jakahira-tohoku@umin.ac.jp

K. Tanoguchi

Department of Obstetrics and Gynecology,

Sendai Kosai Hospital, Sendai, Japan

\section{Introduction}

Mature cystic teratoma is the most common germ cell tumor of the ovary in young women. It is composed of adult type tissue components of various ontogenic organs, such as epidermis, adipose tissue, cartilage and, rarely, thyroid follicles. The benefit of laparoscopic surgery for the management of mature cystic teratoma is widely recognized, since it decreases postoperative pain, time spent in the hospital and the overall recovery period [1]. However, concerns have been raised about the increased risk of spillage of the teratomaous component into the peritoneal cavity during this kind of surgery [2].

Peritoneal strumosis, a condition in which benign thyroid tissue spreads to the peritoneal cavity, is rare. Several authors have reported 'peritoneal strumosis' at the time of surgery for struma ovarii, in which most cases were finally diagnosed as malignant struma ovarii or papillary/follicular thyroid carcinoma of the ovary [3]. The case of real peritoneal strumosis, where both peritoneal implants and primary ovarian tumor are purely benign, seems to be extremely rare.

In this report, we describe a case of peritoneal strumosis whose peritoneal implants of thyroid tissue were considered purely benign. She underwent laparoscopic surgery for mature cystic teratoma and spillage of content occurred during surgery, 10 years prior to this condition.

\section{Case report}

A 64-year-old woman was referred to our hospital due to a suspicion of a recurrence of rectal carcinoma in September 2011. She received endoscopic surgery for adenocarcinoma in an adenoma of the rectum in February 2010, during 
which local recurrence was pointed out. A thorough examination by computed tomography (CT) revealed multiple small nodules in the pelvic cavity and mesentery (Fig. 1). These nodules were not enhanced by positron emission tomography (PET). Blood examination including tumor markers (CEA and CA19-9) and thyroid functions were within normal limits. Under the diagnosis of recurrence and peritoneal dissemination of rectal carcinoma, she underwent laparoscopic surgery in October 2011. On laparoscopy, several small whitish nodules, $2-3 \mathrm{~cm}$ in diameter, were identified. The nodules were localized in tissue of the pelvic cavity including in the uterus, rectum and mesentery (Fig. 2). No accumulation of ascites was found, nor was there evidence of uterine or left ovarian tumor. After intraoperative diagnosis of benign thyroid tissue was made by frozen section for one nodule, only a sampling biopsy was performed. Her postoperative course was uneventful and she has remained alive with disease for 17 months without additional treatment.

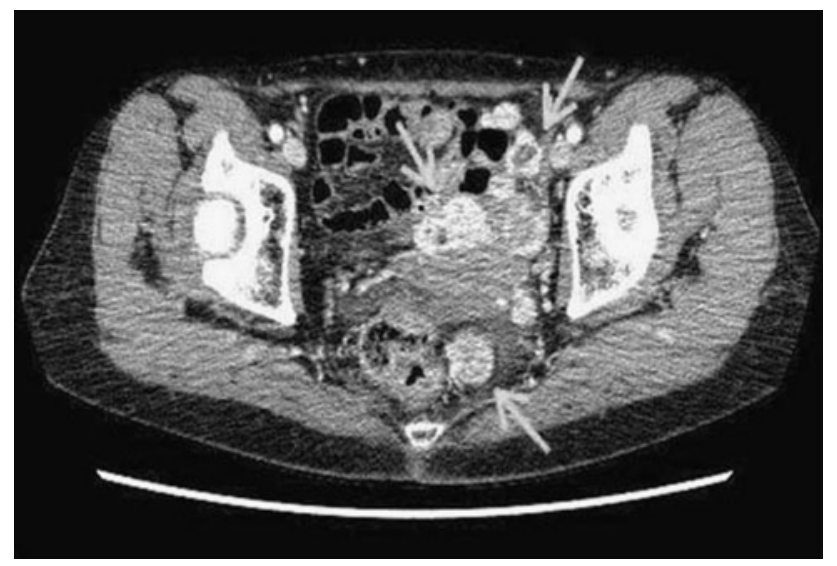

Fig. 1 Image by computed tomography (CT). Multiple small nodules identified in the pelvic cavity and mesentery (arrows)

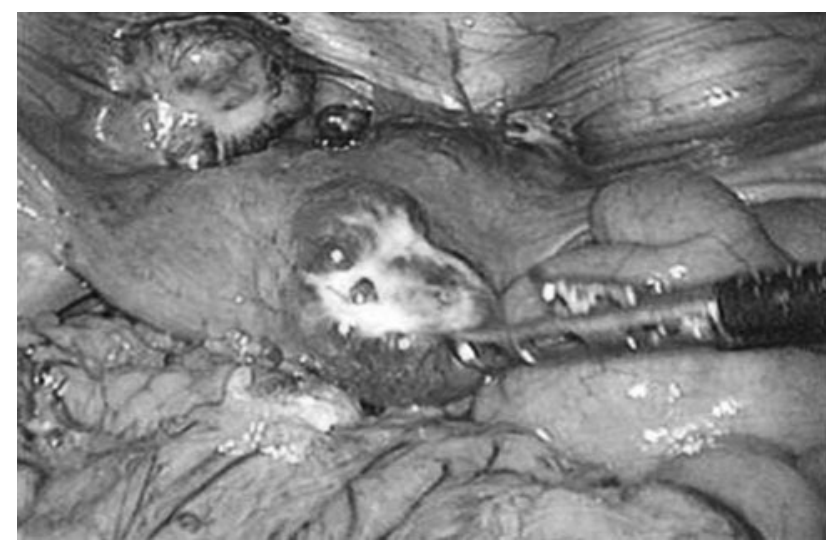

Fig. 2 Macroscopic finding at laparoscopic surgery. Whitish nodules were identified in the uterus and pelvic cavity at the laparoscopic surgery

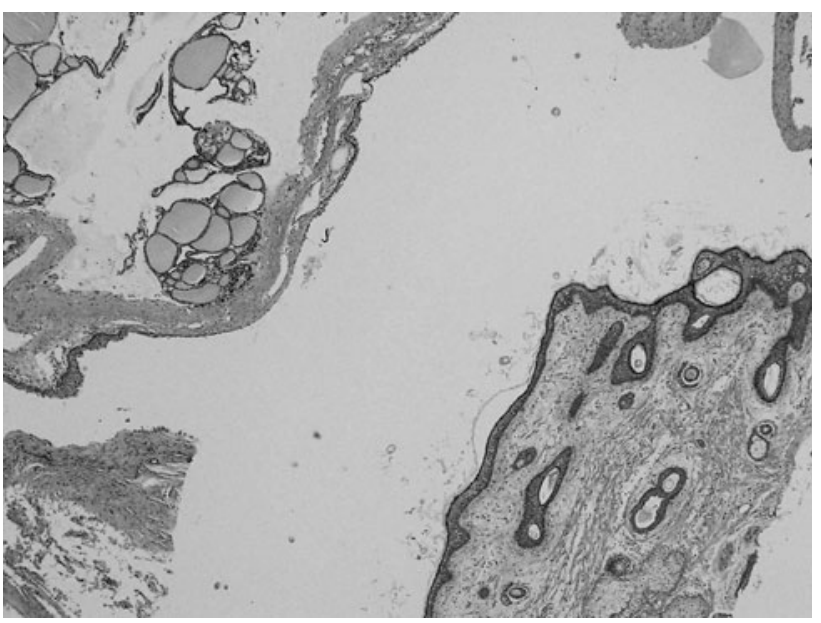

Fig. 3 Histological findings of initial surgical specimen. The right ovarian tumor was composed of teratomatous tissue including dermoid (right) and benign thyroid (left) tissue

After surgery, her past history was re-examined. It was remarkable that she had received a laparoscopic salpingooophorectomy for a right ovarian tumor at another hospital, 10 years prior to present surgery. During the laparoscopic surgery, spillage of fatty component was recorded. Pathological diagnosis was made of mature cystic teratoma with a component of thyroid tissue (Fig. 3).

\section{Pathologic findings}

The excised surgical specimen was fixed in $10 \%$ formalin and embedded in paraffin. $2 \mu \mathrm{m}$-thick sections were prepared and routinely stained with hematoxylin and eosin. Further sections were examined for immunohistochemistry using a streptavidin-biotin amplification method (Nichirei, Tokyo, Japan). Antibodies for the following antigens were used: thyroglobulin (rabbit polyclonal, DAKO Japan Inc., Tokyo, Japan), thyroid transcription factor-1 (TTF-1: mouse monoclonal, 1:100, DAKO Japan Inc.), and Ki67 (mouse monoclonal, 1:100, DAKO Japan Inc.). All procedures have been approved by the Ethics Committee of Sendai Kousei Hospital, and the required informed consents were obtained.

On microscopic examination, the tumor was found to be composed of thyroid follicles of variable size resembling nodular goiter of the thyroid gland (Fig. 4). Nuclear features of papillary thyroid carcinoma, such as nuclear groove or pseudo-inclusion and structural features of papillary or follicular carcinoma, were not identified.

Immunohistochemical staining with anti-thyroglobulin antibody showed strong positive staining in the cytoplasm of the follicular cells. They were also positive for TTF-1. Labeling index of Ki-67 was extremely low $(<1 \%)$. 


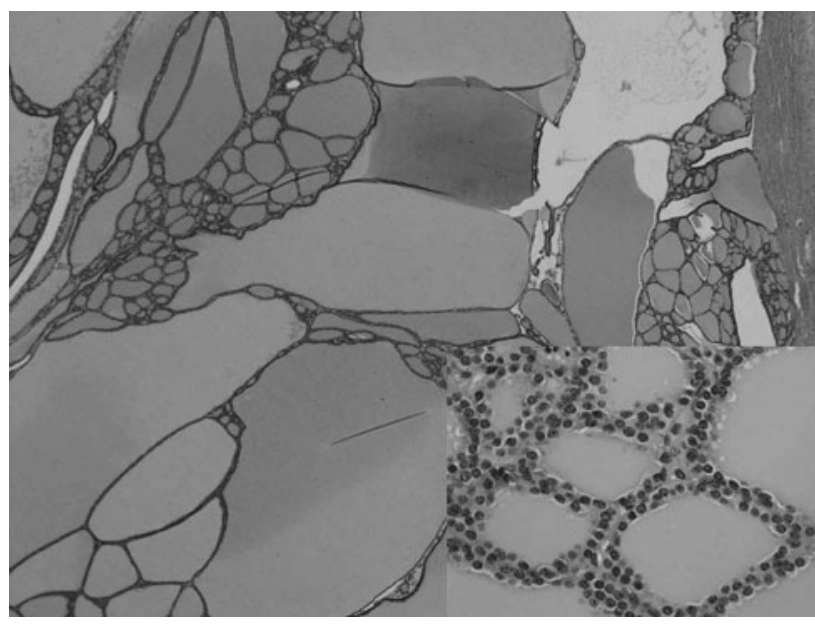

Fig. 4 Histological findings of secondary surgical specimen. Implant nodules on the peritoneum composed of only thyroid tissue with variable size resembling a nodular goiter. No evidence of nuclear features of papillary thyroid carcinoma identified

From these morphological and immunohistochemical results, the patient was finally diagnosed as having peritoneal strumosis.

\section{Discussion}

To our knowledge, the present case is the first case of peritoneal strumosis occurring after treatment with laparoscopic surgery for ovarian mature cystic teratoma. The cause of peritoneal strumosis in this case is strongly suspected as the spillage of the thyroid tissue component during the initial laparoscopic surgery. Implantation and multiple growth of benign thyroid tissue to the peritoneum might have occurred during the decade-long course.

Norris and O'Connor [4] report that, very infrequently, peritoneal implants of benign thyroid tissue, termed 'strumosis', have occurred in struma ovarii and should not be confused with malignancy. Thorough review of literature revealed only 20 reported cases of peritoneal spread of thyroid tissue. Most cases were identified incidentally at the time of initial surgery for ovarian tumor. Roth et al. [3] described in detail the cases of peritoneal implantation or dissemination of thyroid tissues. In their report, they deemed that only two out of 18 cases, including their own, involved purely benign tumors. Four cases were frankly malignant because of distant metastasis, other cases had benign peritoneal dissemination. They concluded a difficulty in deciding whether these tumors are benign or not, because in most cases, the follow-up period was too short. They also proposed considering highly differentiated follicular carcinoma of ovarian origin as a new entity, which resembles thyroid goiter but causes metastasis [3]. In this context, some cases in which peritoneal dissemination was found at the time of surgery for ovarian tumor seems to be malignant struma ovarii, and the term 'peritoneal strumosis' should be used only in cases in which the primary ovarian tumor is purely benign. In our case, the ovarian tumor at the time of the first laparoscopic surgery was an ordinary mature cystic teratoma with a component of the thyroid gland. There was no evidence of malignancy in all of the histological components of her tumor. Also, the peritoneal tumor involved in the secondary surgery was also composed of definitely benign thyroid glands. Thus, we consider the present case as a 'pure' peritoneal strumosis.

In recent years, the laparoscopic approach has greatly improved the treatment of mature cystic teratoma $[2,5]$. The benefits of laparoscopy over laparotomy in the management of benign ovarian cysts include decreased postoperative pain, less time spent in the hospital, and a shorter recovery period [1]. However, laparoscopic surgery for mature cystic teratoma of the ovary has raised concerns about the associated increased risk of tumor content spillage into the peritoneal cavity. In several reports, the rate of the spillage was reported between 12 and $88 \%[2,5,6]$. Cases of chemical peritonitis with subsequent extensive adhesion formation and granulomatosis have been reported following intraperitoneal rupture of mature cystic teratoma [7], although in most cases, complications with spillage was substantially minimal when this was followed by generous irrigation of the abdominal cavity [8]. Spillage of malignant cells following rupture of a teratoma with malignant components raises a more serious problem [9]. In the present case, no malignant component in the primary ovarian tumor identified and no complications like peritonitis were recorded. However, the patient suffered from peritoneal strumosis 10 years after an initial laparoscopic surgery. Thus, we consider the peritoneal strumosis, in other words, as an implantation of benign teratomatous elements to the peritoneum resulting as a delayed complication of intraoperative spillage during laparoscopic surgery. The clinician should treat this tumor with appropriate caution and keep peritoneal strumosis in mind for the differential diagnosis of late complications after laparoscopic surgery.

Conflict of interest The authors declare no conflicts of interest.

\section{References}

1. Lin P, Falcone T, Tulandi T (1995) Excision of ovarian dermoid cyst by laparoscopy and by laparotomy. Am J Obstet Gynecol 173:769-771

2. Kavallaris A, Mytas S, Chalvatzas N et al (2010) Seven years' experience in laparoscopic dissection of intact ovarian dermoid cysts. Acta Obstet Gynecol Scand 89:390-392 
3. Roth LM, Karseladze AI (2008) Highly differentiated follicular carcinoma arising from struma ovaii: a report of 3 cases, a review of the literature, and a reassessment of so-called peritoneal strumosis. Int J Gynecol Pathol 27:213-222

4. Norris HJ, O'Connor DM (1992) Pathology of malignant germ cell tumors of ovary. In Coppleson, M ed. Gynecologic oncology: Fundamental Principles and Clinical Practice. 2nd edn. Churchill Livingstone, London, p 917

5. Zenetta G, Ferrai L, Mignini-Renzini M et al (1999) Laparoscopic excision of ovarian dermoid cysts with controlled intraoperative spillage. Safety and effectiveness. J Reprod Med 44:815-820

6. Kocak M, Dilbaz B, Ozturk N et al (2004) Laparoscopic management of ovarian dermoid cysts: a review of 47 cases. Ann Saudi Med 24:357-360
7. Shamshirsaz AA, Shamshisaz AA, Vibhakar JL et al (2011) Laparoscopic management of chemical peritonitis caused by dermoid cyst spillage. JSLS 15:403-405

8. Campo S, Garcea N (1998) Laparoscopic conservative excision of ovarian dermoid cysts with and without an endobag. J Am Assoc Gynecol Laparosc 5:165-170

9. Mayer C, Miller DM, Ehlen TG (2002) Peritoneal implantation of squamous cell carcinoma following rupture of a dermoid cyst during laparoscopic removal. Gynecol Oncol 84:180-183 